\title{
Epidemiology training: a necessity for primary health
} care

\author{
USHA SHAH \\ From the Department of Preventive and Social Medicine, BJ Medical College, Pune, Maharashta, India
}

SUMMARY In spite of planned development and expansion of health services, especially in rural areas in India, mortality due to preventable conditions has remained unacceptably high. Important reasons for failure of the health system to detect the problems early are centrally planned programmes based on inadequate data and time-bound numerical targets for achievement. This promotes a tendency for passive implementation which destroys initiative and incentive for conceptualisation of problems and strategies. For health programmes to be far more successful, the entire health team needs to be given training in epidemiology appropriate to the level of each category, so that programme planning based on epidemiologically determined local needs can beo done at district level by the District Health Organisation. Participation of health workers and the community will then be more active and relevant to the needs of the community.

In India, one child dies every 6 seconds. Over the past 10 years the infant mortality rate has remained around 125 per 1000 live births. Most of this mortality is due to preventable conditions such as malnutrition and infectious diseases. Thousands of children become blind every year due to vitamin A deficiency. The incidence of malaria is increasing sharply. There is no appreciable change in the prevalence of tuberculosis and leprosy.

To rectify this unsatisfactory state of affairs in spite of planned development and expansion of health services, especially in rural areas, the scheme of village level community health workers was introduced. It was hoped to improve and strengthen the scope of health services through

(a) improving community participation in health care,

(b) demystifying health issues, and

(c) making communities self reliant.

Six years later, in 1984, we face alarming situations produced by large scale epidemics such as hepatitis in Gujerat, dysentery in West Bengal, and recurrent encephalitis-like illness in Maharashtra. These epidemic situations were not appreciated until they had caused several hundred deaths.

One of the most important reasons for the failure of the system to detect these problems early is inadequate feed-back from peripheral levels.
Intelligence from the periphery depends on the $\stackrel{\odot}{\infty}$ efficiency of the grass root and middle level health $\frac{D}{0}$ personnel. This efficiency in turn is determined by the training received and skills developed by them.

In the present era of health for all through primaryo health care, total coverage of defined populations,? community participation, and use of appropriate technologies are basic strategies. These strategies cannot succeed with centrally planned programmes based on inadequate data of questionable reliability, or if programmes are undertaken because specific funding from donor agencies is available. Almost no modifications to suit the local conditions and needs are then attempted. Biases of officials at the central level also play a major role.

Health functionaries are given a list of activities to be performed routinely. The relevance of these activities to the health of the community is rarely explained. Usually time bound targets are given to each member as well as to the health centre. In such situations the overburdened field workers carry out those activities that are physically easiest to perform. Impact on the health status of the community is not therefore perceptible.

For the three tier system of primary health care to be successful, the primary health centre must be the hub of activity. That is where conceptualisation of what primary health care means for that area and what needs to be done to pursue it must be decided. 
In the three tier network, community health workers are the eyes and ears of the health services. Being members of the local community, they come to know of significant or untoward events through personal contacts or local grape-vines. This advantage can be exploited for

(a) routine reporting of specific events such as births and deaths,

(b) surveillance for common communicable diseases such as measles, diarrhoeal diseases, poliomyelitis, etc., and

(c) encouraging health promoting behaviour, eg, appropriate weaning practices, feeding of under-5s, availability of antenatal care and immunisation facilities, personal and environmental hygiene, etc.

Their training therefore needs to stress what to look for, where to look, when and whom to report to, and health reasons for these actions.

Local situations and routinely collected data can be used to explain the basic epidemiological concepts such as susceptible populations, active interventions to decrease the proportions of susceptibles in the community, universal coverage, identification of "at risk" individuals, etc. Similarly, local outbreaks of communicable diseases can be used to stress the importance of early detection and prompt treatment to reduce mortality.

Community health workers can be trained to use simple technologies for screening purposes. It helps to increase their self confidence and credibility in the community. It also increases the involvement of the people and their participation. Another important advantage is that it helps to bring into focus for the community health workers problems such as anaemia, vitamin A deficiency, etc. As a result, their commitment for implementation of preventive interventions is strengthened.

A limited number of nurses are avilable for rural health work. As a result each nurse is assigned to provide services to a specific population around her headquarters. Though she provides preventive services, the emphasis continues to be on treating individuals for minor ailments. With proper training in epidemiology she can be taught to interpret the types of cases she sees in terms of community problems and to bring these to the notice of her supervisors. The training can also give her the ability to judge which responsibilities should be given priority, to supervise and guide the community health workers more effectively and to offer intelligent feed-back to the medical office which would help in programme implementation.

Medical officers of primary health centres see their role primarily as clinicians offering curative services which gives them special status in the community. Preventive and promotive activities are relegated to the paramedicals. In priority programmes such as family planning they mechanically follow instructions issued by the central authority as they are required to submit regular reports of these activities.

In our rural training centre the community health workers were actually involved in basic data collection. We started with simple reporting of births and deaths. The importance of being specific about still births or live births, especially in case of early neonatal death, was explained, and a system to report each event soon after its occurrence was devised. This not only resulted in complete and reliable records but also created a new awareness of where and how the babies were dying. Similarly the use of Salter spring balances to record birthweights prompted the community health workers to give more attention to low birthweight babies and to consider their survival as a personal challenge.

We used Rose Bengal and Kajal tests for screening primary school children for eye signs of vitamin A deficiency. Community health workers did the screening to refer cases to the ophthalmologist. They were staggered to see the high proportion of children with positive signs, who may become blind if neglected. Their concern was communicated to the parents and local leaders who pledged full support to the health centre for reducing that risk. While talking to mothers community health workers stressed foods rich in vitamin $A$ in the daily diet.

Similarly, the community health workers were taught to screen pregnant women for anaemia using the CuSO specific gravity method. They classified mothers in three groups for appropriate intervention:

(a) those with $\mathrm{Hb}$ of $10 \mathrm{~g}$ or more were given iron folic acid supplement and nutrition education;

(b) those with $\mathrm{Hb}$ between 8 and $10 \mathrm{~g}$ were taken to the nurse for further investigation and management decision;

(c) those with $\mathrm{Hb}$ less than $8 \mathrm{~g}$ were considered as high risk cases to be taken straight to the medical officer.

This routine practice by the community health workers made the entire community conscious of the problem. As a result the programme got a boost, and those in urgent need received the care they needed.

A volumetric method using a suitably marked translucent plastic glass was developed for a diet survey. Community health workers did the survey by house to house visits. The results were discussed with them. They were thus able to understand how deficiencies in intake occur and could suggest remedial correction in the diet of the family. To the 
community this was more relevant than theoretical nutritional advice given by paramedicals.

In this area, guinea worm was not reported for many years after systematic eradication efforts by a very eminent community health expert. A couple of cases casually mentioned by staff prompted an investigation. The village from which the cases came was on a seasonal migration route of nomadic shepherds. These nomads spend part of the year in an area known to be endemic for guinea worm. It was soon discovered that some children had removed the barrier to the steps of a nearby well and had used it for swimming. The village leaders appreciated the risk of guinea worm for the entire area, closed the well immediately, and stepped up chlorination of the water. No further cases from that area were reported.

In priority programmes such as MCH/FP, better impact can be achieved if the target group is selected on epidemiological considerations, rather than numerical targets to be achieved. Unfortunately, the current stress on centrally decided, time bound numerical targets promotes a tendency for passive implementation which destroys initiative and incentive for conceptualisation of problems and strategies.

Our experience in the field clearly supports the hypothesis that primary health care programmes can be far more successful if the entire team has a strong epidemiological foundation. Even the community health workers must be given training in epidemiology suitable to their level of functioning.
Unfortunately, very little credit is usually given to the villagers' intelligence, sound common sense, and power of observation. Understanding epidemiological concepts is considered to be beyond their scope.

Currently, epidemiology occupies a minor position in the medical curriculum and is conspicuously absent from the training of other categories of paramedical personnel.

It is necessary to rectify this situation by devising training activities appropriate to each level. This can start with descriptive epidemiology at lower levels, leading to analytical and experimental epidemiology at the physician level. The ability to think in terms of denominators can help the personnel to reach these goals more rapidly.

The training must be non-formal for community health workers and increase in formal format with each level of technically trained individuals to become sophisticated enough to enable the physicians to determine local needs, plan services to address these needs and evaluate the impact on health status. They must also acquire the flexibilityc needed to meet changing situations and emergencies.

Adequately trained epidemiologists are a rarity in most developing countries. The IEA in collaboration市 음 with international funding agencies can take a lead in $\frac{\overrightarrow{\mathrm{D}}}{-}$ conducting pilot projects to demonstrate the need for $\mathrm{c}$ and usefulness of epidemiology training for the entire $\frac{\mathbb{O}}{0}$ team but most essentially for community healtho workers and nurses. 